\title{
EDITORIAL Oncolytic viruses: focusing on the tumor microenvironment
}

Cancer Gene Therapy (2015) 22, 169-171. doi:10.1038/cgt.2015.11

Recent developments in cancer immunotherapy have generated renewed interest in oncolytic viruses as platforms for the development of anti-tumor immunotherapy regimens. Several reviews have detailed exciting new approaches in this area, as well as emphasized the potential success of such agents as part of combinatorial treatment strategies. ${ }^{1,2}$ Indeed, we agree that this treatment modality will most likely achieve its greatest potential as part of combination therapeutic anticancer regimens. However, we would emphasize that the most successful oncolytic virusbased treatments, as well as any combinatorial strategies into which they are incorporated, will likely be those that take advantage of modifying the tumor microenvironment. We and others have used recombinant poxvirus and herpesvirus vectors in both preclinical and clinical studies to demonstrate that oncolytic viruses can be used to modify the cytokine and antigen milieu of the tumor microenvironment, ultimately promoting systemic tumor-specific immune responses that overcome immune escape mechanisms.

A central hypothesis of our studies has been that in situ treatment with oncolytic viruses can be utilized to release an autologous tumor antigen into the tumor microenvironment, thus inducing a systemic tumor-specific immune response against both primary and metastatic tumor. This strategy effectively relies on autologous tumor as a source of multiple antigens, allowing development of a multivalent tumor-specific immune response. Support for this approach can be found in studies that demonstrate an average of 90 mutations per tumor in human breast and colorectal cancers. ${ }^{3}$ Furthermore, it is believed that the immunogenic cell death in the tumor microenvironment that occurs because of oncolytic virus infection triggers release of damage-associated molecular pattern and pathogen-associated molecular pattern molecules, as well as inflammatory cytokines, that aid in the activation and recruitment of antigen presenting cells, ultimately yielding a Th1 immune response. ${ }^{4}$

An essential factor in such immune therapeutic strategies is the idea that the tumor microenvironment acts as the center for tumor immune escape and regulation of anti-tumor immune responses (Figure 1a). Multiple cytokines produced by tumor, particularly IL-10, can have pivotal roles in suppressing the immune response., 5 Studies by our group demonstrated that tumor-induced IL-10 can prevent the induction of both tumor-specific and poxvirus-encoded antigen-specific type 1 immune responses at the tumor site. ${ }^{7}$ Furthermore, at least part of this effect was due to suppression of dendritic cell maturation, thus preventing antigen presentation. ${ }^{6}$ Immune suppressive cytokines, such as IL-10 and TGF- $\beta$, also attenuate cytotoxic T-cell (CTL) function and the Th1 response. ${ }^{8,9}$ The tumor microenvironment promotes the development of suppressive cell populations such as regulatory $T$ cells and myeloid-derived suppressor cells. Both cell populations have inhibitory effects on T-cell function and infiltration into the tumor microenvironment. ${ }^{9-11}$

Given these and other mechanisms of immune escape, we hypothesize that the immune population of the tumor microenvironment can be advantageously adapted by using modified poxviruses to deliver immune stimulatory cytokines, while still taking advantage of the aforementioned direct oncolytic effects of poxviruses. As reviewed by Lichty et al., ${ }^{1}$ oncolytic viruses have been modified to encode numerous immune enhancing cytokines, including IL-2, IL-12 and interferon gamma. We focused our initial efforts on granulocyte macrophage colony-stimulating factor (GM-CSF), based on this cytokine's important role in recruitment and activation of antigen presenting cells. We carried out a phase I trial of intralesional vaccinia-GM-CSF in patients with refractory melanoma. This led to rejection of injected lesions with infiltration of CD4+ and CD8+ T lymphocytes. Moreover, four of seven patients exhibited regression of untreated tumors, suggesting development of a tumor-specific immune response. ${ }^{12}$ This agent, studied as JX-594, has been further demonstrated to preferentially replicate in the tumor microenvironment following intravenous administration in both preclinical and clinical studies. ${ }^{13-15}$ Further evidence supporting this approach was found in subsequent phases I and II clinical trials evaluating an oncolytic herpes simplex virus expressing GM-CSF, which demonstrated that intratumoral injection with this oncolytic virus in melanoma patients resulted in generation of systemic tumor antigen-specific T-lymphocyte responses, as well as decreases in regulatory T cells, suppressor CD8+ T cells and myeloid-derived suppressor cells in patients that developed clinical responses. ${ }^{16,17}$

A complimentary approach which we and others are actively exploring is to utilize oncolytic viruses as vectors for expression of tumor-associated antigen (TAA). Further supporting our central hypothesis that oncolytic viruses can be used as tools to modify the immunological components of the tumor microenvironment, we found in the MB49 murine model of bladder cancer that delivery of recombinant vaccinia virus expressing the immunodominant major histocompatibility complex class I epitope of the TAA overcame systemic anergy and resulted in a systemic CTL response. ${ }^{18}$ The Schlom group independently arrived at similar conclusions in a transgenic colon cancer model using intratumoral subcutaneous priming and intratumoral boosting with recombinant vaccinia virus expressing the carcinembryonic antigen (CEA). ${ }^{19}$ Most recently using our NBT1 orthotopic breast cancer model we found that TAA addition resulted in reduction of myeloid-derived suppressor cell in the tumor and periphery, induction of a systemic T-cell response and tumor regression. ${ }^{20}$ It is possible that the effectiveness of intratumoral oncolytic virus expressing TAA involves multiple mechanisms, including tumor oncolysis, improved TAA presentation by antigen presenting cells leading to a systemic TAA-specific immune response, and increased antigen expression by infected tumor cells enabling improved CTL-mediated killing (Figure 1b). Lee et al. ${ }^{21}$ found that addition of cisplatin to intratumoral vaccinia expressing TAA resulted in increased $C D 11 c+$ dendritic cells in the tumor and increased antigen-specific CD8+ T cells systemically, further supporting the critical role that antigen presentation in the tumor microenvironment likely plays in generation of tumor-specific T-cell responses from intratumoral vaccination with oncolytic vaccines expressing TAA. The finding of increased inflammatory and T-cell infiltration in both local and distant lesions after intratumoral treatment with Newcastle disease virus, and subsequent systemic response to CTLA-4 blockade, shows the critical role that the inflammatory mileu induced by oncolytic virus itself likely plays in attracting anti-tumor $\mathrm{T}$ lymphocytes. ${ }^{22}$ Clinical studies support the advantages of intratumoral poxviruses expressing TAA, including ongoing clinical trials of intraprostatic 

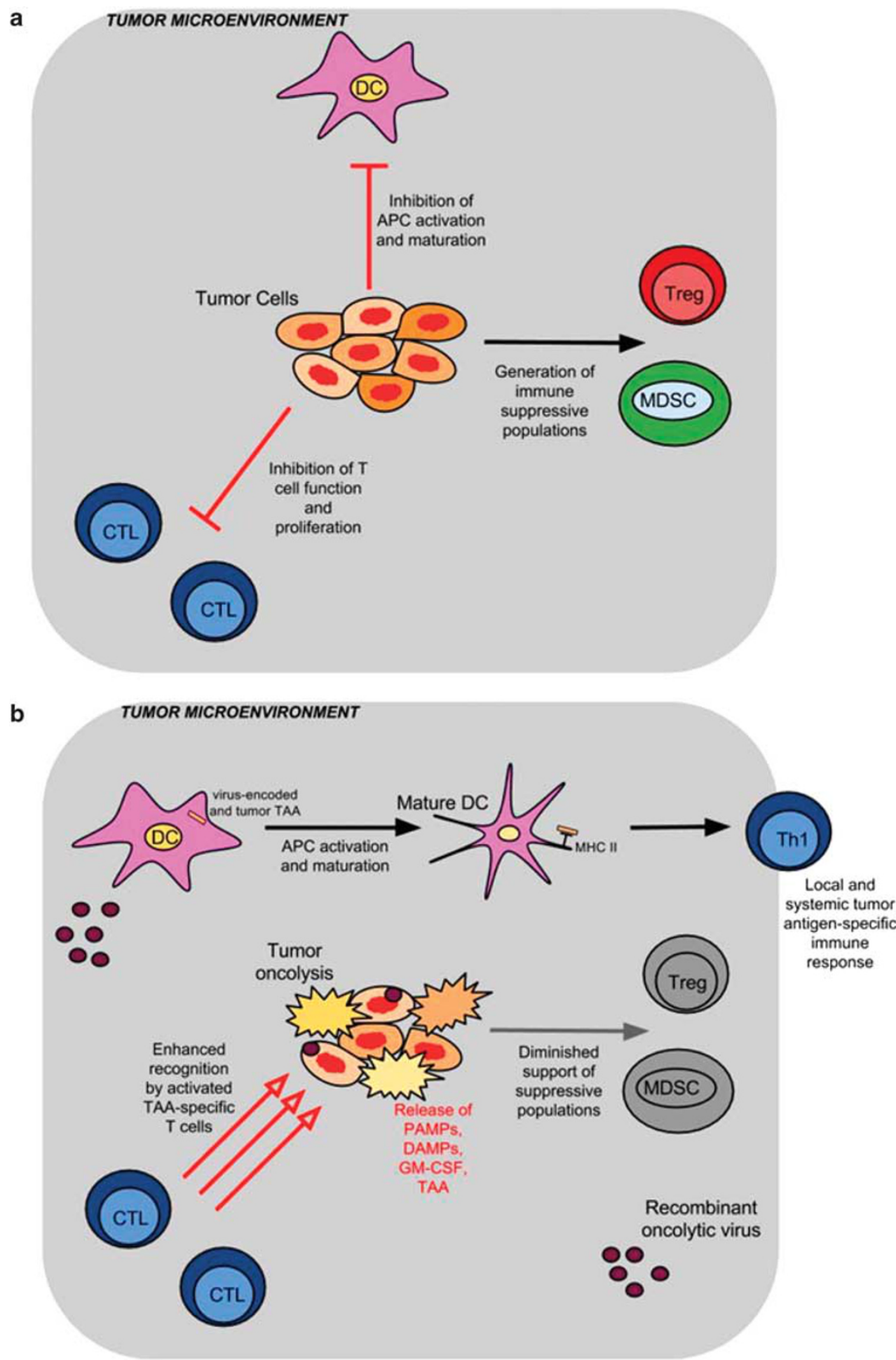

Figure 1. Tumors actively employ several immune escape mechanisms, including interference with antigen presentation, inhibition of T-cell function and proliferation and induction of immune suppressive cell populations such as myeloid-derived suppressor cells and regulatory T cells (a). Oncolytic viruses expressing immune stimulatory cytokines and/or tumor-associated antigen likely overcome immune escape via multiple mechanisms. Tumor oncolysis leads to release of pathogen-associated molecular patterns, damage-associated molecular patterns and cytokines and antigen encoded by the oncolytic virus. This likely leads to a better trafficking and function of antigen-specific cytotoxic T cells. Improved maturation and antigen presentation by antigen presenting cells induce a tumor-specific systemic immune response. The modified immune cytokine milieu results in less support for myeloid-derived suppressor cells and regulatory T cells (b).

poxviruses expressing prostate-specific antigen in patients with prostate cancer ${ }^{23,24}$ and our recently concluded phase I trial of intratumoral Panvac in patients with locally advanced pancreatic cancer. $^{25}$
Recombinant oncolytic viruses, expressing both TAA and immune stimulatory cytokines represent a powerful tool for transforming the tumor microenvironment to support an improved antigen presentation and generation of systemic immune 
responses specific to immunogenic TAA. We predict that this approach, in combination with traditional and devloping systemic therapies, will yield improved outcomes in cancer immunotherapy.

\section{CONFLICT OF INTEREST}

Dr Lattime is an inventor of the patented recombinant Vaccinia-GMCSF which has been licensed to Sillajen and is being studied as Pexa-Vec (JX-594). As such, he derives royalties and licensing fees from Thomas Jefferson University where the patent is held. Dr Howard Kaufman has a consulting/advisory role to Alkermes, Amgen, Merck, Merck Serono, Promethus, and currently has funding from BristolMeyers Squibb/Medarex. Dr de Vries has no conflict of interest.

CR de Vries, HL Kaufman and EC Lattime

Rutgers Cancer Institute of New Jersey and Department of Surgery,

Rutgers Robert Wood Johnson Medical School, Rutgers, The State University of New Jersey, New Brunswick, NJ, USA

Correspondence: Dr EC Lattime, Rutgers Cancer Institute of New Jersey and Department of Surgery, Rutgers Robert Wood Johnson Medical School, Rutgers, The State University of New Jersey, 195 Little Albany Street, New Brunswick, NJ 08903, USA. E-mail: e.lattime@rutgers.edu

\section{REFERENCES}

1 Lichty BD, Breitbach CJ, StojdI DF, Bell JC. Going viral with cancer immunotherapy. Nat Rev Cancer 2014; 14: 559-567.

2 Guo ZS, Bartlett DL. Oncolytic viruses as platform for multimodal cancer therapeutics: a promising land. Cancer Gene Ther 2014; 21: 261-263.

3 Sjoblom T, Jones S, Wood LD, Parsons DW, Lin J, Barber TD et al. The consensus coding sequences of human breast and colorectal cancers. Science 2006; 314: 268-274.

4 Donnelly OG, Errington-Mais F, Steele L, Hadac E, Jennings V, Scott K et al. Measles virus causes immunogenic cell death in human melanoma. Gene Ther 2013; 20: 7-15.

5 Sato T, Terai M, Tamura Y, Alexeev V, Mastrangelo MJ, Selvan SR. Interleukin 10 in the tumor microenvironment: a target for anticancer immunotherapy. Immunol Res 2011; 51: 170-182.

6 Yang AS, Lattime EC. Tumor-induced interleukin 10 suppresses the ability of splenic dendritic cells to stimulate CD4 and CD8 T-cell responses. Cancer Res 2003; 63: 2150-2157.

7 Halak BK, Maguire HC Jr., Lattime EC. Tumor-induced interleukin-10 inhibits type 1 immune responses directed at a tumor antigen as well as a non-tumor antigen present at the tumor site. Cancer Res 1999; 15: 911-917.

8 Takaku S, Terabe M, Ambrosino E, Peng J, Lonning S, McPherson JM et al. Blockade of TGF-[beta] enhances tumor vaccine efficacy mediated by CD8+ T cells. Int J Cancer 2010; 126: 1666-1674.

9 Strauss L, Bergmann C, Szczepanski M, Gooding W, Johnson JT, Whiteside TL. A unique subset of CD4+CD25highFoxp3+ T cells secreting interleukin-10 and transforming growth factor-beta1 mediates suppression in the tumor microenvironment. Clin Cancer Res 2007; 13: 4345-4354.

10 Lechner MG, Megiel C, Russell SM, Bingham B, Arger N, Woo T et al. Functional characterization of human $\mathrm{Cd} 33+$ and $\mathrm{Cd} 11 \mathrm{~b}+$ myeloid-derived suppressor cell subsets induced from peripheral blood mononuclear cells co-cultured with a diverse set of human tumor cell lines. J Transl Med 2011; 9: 90.

11 Lesokhin AM, Hohl TM, Kitano S, Cortez C, Hirschhorn-Cymerman D, Avogadri F et al. Monocytic CCR2(+) myeloid-derived suppressor cells promote immune escape by limiting activated CD8 T-cell infiltration into the tumor microenvironment. Cancer Res 2012; 72: 876-886.

12 Mastrangelo MJ, Maguire HC Jr, Eisenlohr LC, Laughlin CE, Monken CE, McCue PA et al. Intratumoral recombinant GM-CSF-encoding virus as gene therapy in patients with cutaneous melanoma. Cancer Gene Ther 1999; 6: 409-422.

13 Park BH, Hwang T, Liu TC, Sze DY, Kim JS, Kwon HC et al. Use of a targeted oncolytic poxvirus, JX-594, in patients with refractory primary or metastatic liver cancer: a phase I trial. Lancet Oncol 2008; 9: 533-542.

14 Parato KA, Breitbach CJ, Le Boeuf F, Wang J, Storbeck C, Ilkow C et al. The oncolytic poxvirus JX-594 selectively replicates in and destroys cancer cells driven by genetic pathways commonly activated in cancers. Mol Ther 2012; 20: 749-758.

15 Breitbach CJ, Burke J, Jonker D, Stephenson J, Haas AR, Chow LQM et al. Intravenous delivery of a multi-mechanistic cancer-targeted oncolytic poxvirus in humans. Nature 2011; 477: 7102-7362.

16 Kaufman HL, DeRaffele G, Divito J, Horig H, Lee D, Panicali D et al. A phase I trial of intralesional $\mathrm{rV}$-Tricom vaccine in the treatment of malignant melanoma. Hum Gene Ther 2001; 12: 1459-1480.

17 Kaufman HL, Kim DW, DeRaffele G, Mitcham J, Coffin RS, Kim-Schulze S. Local and distant immunity induced by intralesional vaccination with an oncolytic herpes virus encoding GM-CSF in patients with stage IIIc and IV melanoma. Ann Surg Oncol 2010; 17: 718-730.

18 Yang $A S$, Monken $C E$, Lattime EC. Intratumoral vaccination with vacciniaexpressed tumor antigen and granulocyte macrophage colony-stimulating factor overcomes immunological ignorance to tumor antigen. Cancer Res 2003; 63: 6956-6961.

19 Kudo-Saito C, Schlom J, Hodge JW. Intratumoral vaccination and diversified subcutaneous/ intratumoral vaccination with recombinant poxviruses encoding a tumor antigen and multiple costimulatory molecules. Clin Cancer Res 2004; 10: 1090-1099.

20 de Vries CR, Monken CE, Lattime EC. The Addition of Recombinant Vaccinia HER2/ neu to Oncolytic Vaccinia-GMCSF Given into the Tumor Microenvironment Overcomes MDSC-Mediated Immune Escape and Systemic Anergy. Cancer Gene Ther 2015; 22: 154-162.

21 Lee SY, Kang TH, Knoff J, Huang Z, Soong RS, Alvarez RD et al. Intratumoral injection of therapeutic HPV vaccinia vaccine following cisplatin enhances HPV-specific antitumor effects. Cancer Immunol Immunother 2013; 62: 1175-1185.

22 Zamarin D, Holmgaard RB, Subudhi SK, Park JS, Mansour M, Palese $P$ et al. Localized oncolytic virotherapy overcomes systemic tumor resistance to immune checkpoint blockade immunotherapy. Sci Transl Med 2014; 6: 226 ra32.

23 Gulley JL, Heery CR, Madan RA, Walter BA, Merino MJ, Dahut WL et al. Phase I study of intraprostatic vaccine administration in men with locally recurrent or progressive prostate cancer. Cancer Immunol Immunother 2013; 62: 1521-1531.

24 Madan RA, Heery CR. Gulley JL. Poxviral-based vaccine elicits immunologic responses in prostate cancer patients. Oncoimmunology 2014; 3: e28611.

25 Repaka A, Poplin EA, August DA, Ben-Menachem T, Michael H, Artymyshyn R et al. Phase I trial of endoscopic ultrasound (EUS) guided intratumoral vacination with recombinant Panvac- $\mathrm{F}$ and stsyemic Panvac- $\mathrm{V}$ in patients with locally advanced pancreatic cancer. Gastrointest Endosc Suppl 2013; 77: AB143. 